\title{
Profesionales del Futuro: La formación por competencias a través de la co-presentación.
}

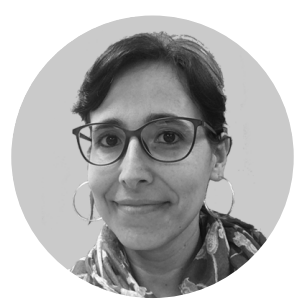

MARIONA GRAELL

UNIVERSITAT INTERNACIONAL DE CATALUNYA

mgraell@uic.es

$\mathrm{E}$ ste artículo presenta una experiencia práctica que permite desarrollar distintas competencias de la asignatura de aprendizaje de las lenguas y lectoescritura III en una sola actividad. En la Universitat Internacional de Catalunya la copresentación propone a los estudiantes unificar distintos saberes para dar respuesta a un reto específico de materia: presentando, analizando y evaluando un trabajo elaborado por otro equipo de estudiantes. Para el desarrollo de esta estrategia metodológica cabe tener en cuenta algunas consideraciones como el contexto, la planificación de la asignatura y la preparación del profesorado universitario en cuestiones participativas para su funcionamiento. Una experiencia universitaria que propone revisar praxis docentes clásicas y complementarlas con otras más activas para una preparación profesional en competencias. De este modo se busca aplicar las competencias de materia, de titulación y las transversales de forma operativa mediante la sistematización de la actividad de copresentación.

\section{PALABARAS CLAVE:}

Innovación docente; capacidad crítica; educación superior; participación; trabajo en grupo. deve his article presents a practical classroom experience that allows developing different competencies of the subject 'Language learning and literacy III' within a single activity. At the Universitat Internacional de Catalunya, the activity of co-presentation affords students the opportunity to use different types of knowledge to respond to a specific challenge stated in the subject: in small groups, to present, analyze and evaluate a project designed by another group. For the correct development of this methodology strategy, it is necessary to take into account some considerations such as the context, the course planning and the need for professional preparation of the university lecturers in terms of the participatory issues involved in such a methodological approach. This university experience proposes to review classic teaching praxis and complement them with more active ones for professional competency-based preparation. In this way, the aim is to apply the competencies from the course, the whole degree and the transversal competences in an efficient way through the systematization of the copresentation activity.

\section{KEYWORDS:}

Teaching innovation; critical ability; higher education; participation; group work. 


\section{Introducción}

L os universitarios a los que formamos hoy desarrollarán sus profesiones de múltiples y diversas formas a las que aún no podemos precisar. Los avances tecnológicos nos dificultan nuestras previsiones, incluso en el mundo educativo en el que todo parecía tener un orden establecido. Los métodos magistrales clásicos preparan a los estudiantes en conocimiento pero no especialmente en saber hacer (Álvarez Suarez et al, 2014). El trabajo por competencias en el sistema universitario europeo (European Ministers Responsible for Higher Education, 2009) nos obliga a repensar nuestras materias, nuestras formas de planificar las asignaturas y sus evaluaciones, pero sobretodo nuestra metodología docente (Cano, 2008). La competencia parte de una perspectiva cognitiva que supone entenderla como capacidades muy amplias (Perrenoud, 2004), que implican elegir y movilizar recursos, tanto personales (conocimientos, procedimientos, actitudes) como de redes (bancos de datos, acceso documental, especialistas,...) y realizar con ellos una atribución contextualizada (espacio, tiempo, relación) (Cano, 2008, p.6). El desarrollo de competencias que promueve el Espacio Europeo de Educación Superior (EEES), también conocido como "plan de Bolonia" se sitúa en un paradigma interpretativo, una concepción holística, en un trabajo complejo que no se resuelve con un algoritmo o protocolo sino que exige iniciativa, transferencia e innovación. Se fomenta una manera de formar ligada al desarrollo profesional y personal más allá de puestos de trabajo concretos, concebida como un "saber actuar", requiriendo una reflexión teórica, para tener presente el propósito y las consecuencias o impactos de las propias acciones.

Ante estas concepciones formativas y la aplicación del llamado "plan de Bolonia", la importancia de innovar en las aulas universitarias es más una necesidad que una opción. Las metodologías únicas quedan obsoletas ante el requisito de formar competentemente a los estudiantes universitarios. Parece que la innovación pasa por la variedad de aproximaciones metodológicas en una misma materia. La pedagogía del siglo XXI requiere de las aulas universitarias la promoción de la evaluación continuada en detrimento de la evaluación única; la necesidad de la participación activa de los estudiantes para poder explorar su saber actuar en pro de la autonomía, el trabajo en grupo, proactividad y la investigación. Preparando así a un profesional dispuesto a aprender a lo largo de la vida de forma continuada teniendo en cuenta que la educación se ha democratizado y es permanente (Estella y Vera, 2008). Este artículo pretende mostrar una propuesta formativa en competencias en el aula universitaria con el fin de integrar los conocimientos de la materia con habilidades necesarias para la profesión docente y el desarrollo profesional. De este modo se presentan, inicialmente, las reflexiones que llevan a la propuesta, así como las cuestiones más organizativas para la puesta en marcha de la experiencia; posteriormente, se relata de forma sistematizada la experiencia y se acaba concluyendo con algunas ideas que puedan llevar a desarrollar nuevas experiencias y reflexiones.

\section{Contextualización}

a formación de maestros de educación infantil en la materia de Aprendizaje de las lenguas y lectoescritura tiene cierto grado de complejidad por ser una actividad compleja y de la que dependen muchas variables. Tratar esta temática desde el aula universitaria clásica resulta insuficiente para el desarrollo profesional del futuro maestro. En la Universitat Internacional de Catalunya (UIC) tratamos de dar respuesta a estos retos desde el trabajo por competencias de la asignatura y la innovación para adaptarnos a los estudiantes de hoy.

La integración de saberes en la formación de los futuros profesionales docentes debe permitir, por una parte la relación entre el conocimiento teórico y el conocimiento práctico y, al mismo tiempo, debe hacer posible la creación de nuevo conocimiento a partir de la práctica (Cochran-Smith, 2002). La formación académica y la formación práctica deben ser complementarias y retroalimentarse de manera continua puesto que una no existe sin la otra (Álvarez Rateike, 2011).

El planteamiento de la asignatura que presentamos tiene como finalidad la adquisición de conocimientos a partir de la práctica, incluyendo métodos y procedimientos adecuados para aprender haciendo (learning by doing) que presupone que el estudiante posea conocimientos teóricos básicos que le permitan acceder a la formación, y conocimientos prácticos que le permitan la reflexionar sobre su actuación (Graell y Fuertes, 2019). Para Schön (1992) la reflexión forma parte de la práctica profesional y apuesta por un aprendizaje más profundo. La experiencia educativa debe permitir a los estudiantes observar, preguntarse, descubrir, analizar, reformular, interpretar, integrar, aplicar, intervenir, comunicar y en definitiva investigar, imaginar y crear.

Por lo tanto, la mera aplicación de los contenidos teóricos resulta insuficiente y es preciso proporcionar conocimientos más ligados a la acción y a la reflexión. Según Schön (1992), lo más preciso sería enseñar a nuestros estudiantes a tomar decisiones bajo condiciones de incertidumbre, pero esto es precisamente lo que más cuesta enseñar desde las aulas de las universidades. La acción en el contexto debe ofrecer la oportunidad para aprender y relacionar los conocimientos asimilados a partir de la implicación personal y la experiencia. Es lo que Marcelo (2009) define como "aprendizaje activo" a partir del desarrollo de proyectos de trabajo profesionales.

\section{Maestros como artesanos}

a experiencia educativa que se presenta aquí tiene como objetivo el trabajo orientado a la calidad - (metafóricamente artesanal) que se contrapone con la mirada educativa de carácter burocrático y de arte orientado al cumplimiento del mandato (Collet-Sabé, 2011). Se plantea la educación como un proceso -artesano- que requiere de lentitud, del trabajo colectivo y cooperativo, desde las manos y la cabeza, es decir, para aprender haciendo. 
Un buen maestro es aquel que se configura en la práctica en el aula, mediante el deseo de educar bien, basándose en los intercambios y el compromiso compartido; la aceptación de la complejidad de educar, que puede presentar dificultades, errores y fracasos; el convencimiento de ser artesano en constante construcción presentándose la realidad como un espacio para entrenar destrezas del aprendiz como si de un taller se tratara; la colectividad como un espacio para sentirse acompañado, para compartir, comprender y aprender; y en el tiempo lento que es el que posibilita la reflexión, deliberación y la capacidad de decisión meditada (Collet-Sabé, 2011, 45). Permitiendo así mantener la exigencia y un alto nivel de aprendizaje.

Mediante esta perspectiva el maestro-artesano necesita de la realidad-taller para construir conocimiento y dar sentido a los saberes teóricos, mediante la experimentación, el acierto, la equivocación y la reflexión. Nuestros estudiantes-maestros noveles necesitan de esta experimentación en su proceso de formación para educar bien. El perfeccionamiento en su destreza docentes es clave pasando de la mano (práctica) a la cabeza (teoría).

\section{Metodologías activas}

E n la universidad hay distintas formas de enseñar el conocimiento académico: mediante el aprendizaje pasivo del estudiante, denominado pasivo porque el protagonismo lo asume el docente, y el aprendizaje activo, en el que el estudiante asume mayor protagonismo en su participación en el proceso de la enseñanza-aprendizaje (Imbernon y Medina, 2005, 8). En las metodologías activas se trata de dar voz al estudiante, que tome consciencia de la responsabilidad de su propio proceso de aprendizaje. Este cambio supone concebir el proceso de enseñanzaaprendizaje, que aparecen en las aulas universitarias, desde una perspectiva bidireccional, en el que tanto el docente como el alumno contribuye mediante ideas y experiencias previas para una construcción de conocimiento conjunto.

El docente cumple una nueva función en este escenario; es el que se encarga de acompañar, dirigir e interpelar al estudiante en la materia que tratan. El estudiante sale de la zona de confort, puesto que se expone a un escenario recóndito, pero el docente también - ya que a partir de ahora ha de procurar que el estudiante desarrolle un conjunto de competencias y no únicamente adquiera conocimientos. No se trata de priorizar el esfuerzo por saber mucho, sino por llegar a ser una persona completa (Martín y Puig, 2007).

En el contexto de la formación universitaria de futuros maestros, la importancia de formar a los estudiantes desde una perspectiva holística posibilita múltiples saberes que se ponen en práctica en el aula y en la realidad. Desde este prisma, se permite problematizar mínimamente la conceptualización de la educación, poniendo en duda el trabajo rápido, aislado, de cabeza, perfeccionista, competitivo, repetitivo, potenciado por la idea de vocación y talento. Las universidades tras esta perspectiva padecen el síndrome de aceleración (Bauman, 2001, 21), en la que la educación se considera como un producto más que como el proceso (Myres, 1960, 262).

Para planificar una metodología activa cabe preparar al estudiante, para que poco a poco vaya tomando mayor autonomía en su proceso de aprendizaje y se sienta capaz de configurar su propia idea desarrollando la capacidad crítica. Es por ello que debe ser una forma de hacer constante en las asignaturas, entrenando a los estudiantes a tener mayor participación, desde técnicas iniciales como la de brainstorming, a discusiones ràpidas o los debates dirigidos hasta los trabajos colaborativos o cooperativos o el aprendizaje servicio (Trilla, 2009). Este tipo de metodologías interceden directamente en cómo se planifican los contenidos, las sesiones y las evaluaciones. Requieren de un acompañamiento constante donde el estudiante va contrastando continuamente sus saberes y sus competencias en una evaluación continuada (Imbernon y Medina, 2005). Con las aportaciones, comentarios y observaciones del docente, el estudiante va reconduciendo sus actuaciones para ir afinando las competencias necesarias para poder ser competente en cuestiones de esa materia (Gibbs y Simpson, 2009).
${ }^{66}$ El maestro-artesano necesita de la realidad-taller para construir conocimiento y dar sentido a los saberes teóricos, mediante la experimentación, el acierto, la equivocación y la reflexión.

Nuestros estudiantesmaestros noveles necesitan de esta experimentación en su proceso de formación para educar bien. El perfeccionamiento en su destreza docentes es clave pasando de la mano (práctica) a la cabeza (teoría)." 


\section{La Planificación del docente}

a planificación normalmente se refiere al diseño $\mathrm{y}$ determinación de las intenciones educativas de la materia, especificando cómo se llevará a cabo mediante actividades dotadas de contenidos. Las actividades han de tener unos objetivos específicos, un proceso metodológico y evaluativo, e identificado y trazado el proceso de aprendizaje que seguirá el estudiante.

Para que el profesorado pueda planificar un buen proceso de aprendizaje del estudiante participante, es recomendable dar respuesta a algunas preguntas que puedan ayudar a reflexionar y analizar sobre la forma en cómo presentaremos y trabajamos el contenido o materia.

Las preguntas que se presentan en la figura $\mathbf{1}$ acompañan a la elaboración del diseño de un programa de asignatura como el de una sesión que se basa en metodologías más participativas, teniendo en cuenta los procesos de aprendizaje que el estudiante ha de seguir para asumir unas competencias que se determinan en la asignatura. Estos interrogantes permiten una mejor preparación de la actividades docentes y a la vez la toma de conciencia de los aspectos sobre los que debería realizarse nuestra acción formativa.

\section{Trabajo cooperativo en equipos de trabajo}

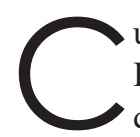

uando planteamos una propuesta educativa desde el EEES es fundamental recordar que las actividades que proponemos han de ayudar a que los estudiantes sean competentes tanto en el contenido propio de la materia como en las habilidades para poderla desarrollarla e implementarla con otros profesionales. Es por ello que se plantea trabajar el contenido a través de una actividad colaborativa y de cooperación en la que los estudiantes se distribuyen en pequeños equipos de trabajo, preferiblemente heterogéneos, para ayudarse y animarse mutuamente a la hora de realizar las actividades del aprendizaje en general (Pujolàs y Lago, 2011, p. 13). Los estudiantes que se implican en una actividad cooperativa pueden vivenciar la ayuda mutua para su desarrollo, compartiendo capacidades, competencias e informaciones que hacen que las soluciones a las cuestiones planteadas, al inicio de la actividad, sean reflexionadas, discutidas e integradas entre todos los miembros del equipo. La investigación aporta evidencias que los estudiantes asumen mayores habilidades y capacidades cuando trabajan conjuntamente que cuanto lo hacen de forma individual (Pujolàs y Lago, 2011).

Es por ello que la propuesta pedagógica que se presenta en este artículo saca de la zona de confort al estudiante pidiéndole en un primer momento que trabaje en equipo de forma colaborativa (Prendes, 2003) empoderándose entre sí.

Según Borràs (en Martínez y Prendes, 2007:28), "el aprendizaje constructivista se caracteriza por la transformación del conocimiento. Aprender no significa simplemente reemplazar un punto de vista (el incorrecto) por otro (el correcto), ni acumular nuevo conocimiento sobre el viejo, sino transformar el conocimiento. Esta transformación, a su vez, ocurre a través del pensamiento activo y original del alumno. Este debe considerar sus saberes previos e implicarse en la experimentación y la resolución de problemas. Considera que los errores no son antitéticos del aprendizaje sino más bien la base del mismo. Por otra parte, se comprende mejor cuando se apoyan, involucran y expanden los propios intereses. En cuanto a las relaciones intra e interpersonales, es fundamental el desarrollo de la autonomía y la interacción de los alumnos entre sí y entre éstos y el docente".

(Martínez y Prendes, 2007, p.28)

\section{La experiencia de aprendizaje de las lenguas y lectoescritura en Educación Infantil de la UIC}

L a asignatura de Aprendizaje de las Lenguas y Lectoescritura -contiene 4 módulos- tiene por objetivo que los futuros maestros sean capaces de enseñar el lenguaje y acompañar a los niños de 0-6 años en la lectura y la escritura, comunicándose oralmente y por escrito $\mathrm{y}$ definiendo con fundamentación teórica su posicionamiento. En concreto, el módulo 3 consta de 6 créditos y tiene como propósito conocer la potencialidad de la literatura infantil en la etapa de educación infantil; identificar los distintos géneros de la literatura infantil, y dominar los recursos para motivar la lectura en la educación infantil.

La metodología de esta materia es variada y activa, demandando a los estudiantes que participen y contribuyan a construir la materia, mediante las lecturas y las explicaciones realizadas en el aula. Las competencias que deberían asumir a lo largo de la asignatura se pueden consultar en el anexo 1.

El desarrollo de la asignatura busca un equilibrio entre diferentes actividades formativas desarrollándose a través del modelo competencial de la pirámide de Miller (1990). Una propuesta docente que contempla (1) una parte cognitiva -con clases teóricas, seminarios y debates (saber) y de trabajos en grupo e individuales (saber cómo aplicar)y (2) una parte conductual -con talleres, dinámicas (saber mostrar) y prácticas a la comunidad (saber hacer).

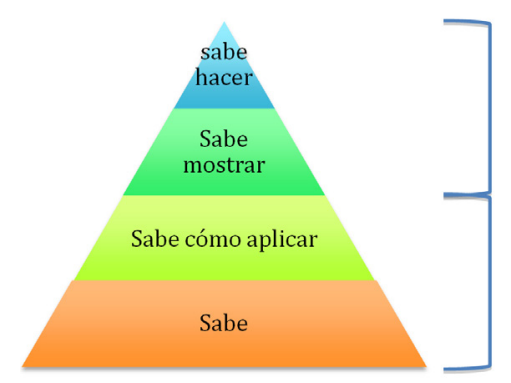

Conductual

Cognitiva

llustración 1. Adaptación de la pirámide de Miller (1990). 


\section{Presentación}

- ¿Tienes claros los objetivos de la clase?, ¿los temas?, ¿los contenidos?, ¿las actividades? y ¿la relación que tienen entre ellos?

- ¿Presentas el tema sobre el que tratarás en clase?, ¿explicas cuál es el objetivo de la sesión?

- ¿Presentas las preguntas en un orden lógico?

- ¿Les dices el tiempo que tienen para hacer cada actividad?

\section{Herramientas para dar la clase}

- ¿Cómo hablas a los estudiantes?

- ¿Cómo usas el lenguaje corporal?

- ¿Utilizas el humor como recurso?

- ¿Hablas a todo el grupo-clase, a grupos particulares, a estudiantes concretos?

- ¿Cómo gestionas el aula?

\section{Estrategias y materiales didácticos}

- ¿Defines con claridad los conceptos y términos que presentas?

- ¿Utilizas ideas anteriores para presentar las nuevas y las relacionas?

- ¿Usas distintas estrategias cuando los alumnos no te entienden?

- ¿Cómo usas la pizarra? ¿tienes alguna estrategia pensada para su uso?

- ¿Qué utilidad le das a los textos escritos? ¿Cómo los integras en la dinámica del aula?

- ¿Qué uso de las TIC haces en el aula? ¿Cómo las introduces en el aula?

- ¿Qué material de elaboración propia haces? ¿Por qué lo haces y cómo tiendes a usarla?

\section{Diálogo, participación y experimentos}

- ¿Cómo facilitas la participación de los estudiantes en el aula?

- Cuando explicas, ¿haces preguntas a los estudiantes?

- ¿Qué tipo de preguntas haces? ¿Cómo les ayudan a pensar?

- ¿Te esperas unos instantes para dejarles responder?

- ¿Reformulas la pregunta si es necesario?

- ¿Qué haces cuando se equivocan?

- ¿Cómo animas a los estudiantes para que formulen preguntas?

- ¿Monopolizas la palabra durante las clases?

- ¿Usas alguna estrategia para que todo el mundo pueda participar?

- ¿Cómo propicias la discusión entre los propios estudiantes?

- ¿Usas el trabajo en grupo y el aprendizaje cooperativo?

- ¿En qué medida puedes decir que tus clases son activas?

\section{Evaluación}

- ¿Les dices el tiempo que tienen para hacer cada tarea?

- ¿Cómo sabes qué conocimientos previos tienen los estudiantes?

- ¿Qué tipo de actividades de evaluación aplicas?

- ¿Devuelves y comentas los resultados de la evaluación?

- ¿Cómo saben los estudiantes que han mejorado?

Figura 1. Preguntas que incitan a la reflexión. Fuente: Martín y Puig, 2008. p.79-82 
En lo que se refiere a la evaluación, tal como lo delimita el EEES, ha de ser continuada con el fin de ofrecer el máximo de posibilidades de aprendizaje y mejora del estudiante, ayudándolo con una retroalimentación continua y manteniendo a su vez $\mathrm{u}+\mathrm{n}$ alto nivel de exigencia. Realiza una doble alimentación:

1 De feedback, el estudiante recibe del docente un retorno constante durante las clases y en la devolución de sus trabajos,

2 De feedforward, en el que los mismos estudiantes acompañados por el docente han de tomar consciencia de lo que han hecho bien, verbalizar y expresar los aspectos que han de mejorar y cómo lo van a hacer. Así, es una herramienta de evaluación que permite por un lado reconocer los aspectos que se han realizado bien ayudando a empoderar al evaluado, y por el otro avanzar en los aspectos que no han salido tan bien (Goldsmith, 2003).

\section{Trabajar mediante la co-presentación}

E n la asignatura de aprendizaje de las lenguas y lectoescritura del grado de educación infantil de la UIC, se lleva a cabo una actividad basado en la copresentación que trabaja las competencias y desarrolla la capacidad crítica de los estudiantes. Esta propuesta pedagógica se centra en dotar a los estudiantes del dominio de recursos para el fomento de la lectura en la educación infantil, en concreto las bibliotecas escolares. Las competencias que se pretenden trabajar en esta actividad se pueden consultar en el anexo 2.

Como se puede observar el volumen de competencias que se determinan es múltiple. Al ser una actividad compleja requiere de la aplicación y entrenamiento de todas estas competencias para que esta propuesta se pueda desarrollar adecuadamente. El objetivo final es que los estudiantes investiguen sobre lo que es una biblioteca escolar, sus características y preparen una propuesta rompedora para medianos del S.XXI tomando como punto de partida las bibliotecas escolares que ven en sus centros de prácticas.

Para ello necesitan:

1 estudiar y revisar el marco teórico de las bibliotecas escolares,

2 compararlo con la realidad de los centros de prácticas de cada uno de los miembros del grupo, y

3 analizar cuáles son las nuevas tendencias literarias, los últimos estudios sobre la promoción de la lectura y el desarrollo cognitivo de los niños así como las preferencias sociales, puesto que el entorno social y cultural de los niños de hoy es distinto a los de hace 20 años (Gaitán, 2010).
El proceso metodológico que se sigue en la actividad dura varias sesiones para posteriormente llegar a la copresentación. Tiene diversas fases que se presentan a continuación.

\section{Primera fase}

\section{Introducción del proyecto}

Para iniciar la actividad se presentan las cuestiones a trabajar, objetivos de aprendizaje y resultados de aprendizaje. En esta ocasión se les otorga a los estudiantes el siguiente encargo hipotético:

"Desde el gobierno se os pide que propongáis el nuevo modelo de biblioteca escolar para las escuelas del S.XXI'.

En esta primera fase el estudiante ha de saber qué competencias se prevé trabajar a lo largo de la propuesta, posibilitando así la toma de conciencia de sus saberes. Al iniciar la actividad cabe despertarles interés y curiosidad para garantizar la participación de todos los estudiantes, es por ello que se les presenta el encargo. Se les explica los tempos de la actividad así como el proceso que se seguirá, primero la realización del trabajo escrito en equipos colaborativos y después la presentación oral en grupo de un trabajo escrito por otro equipo paralelo.

\section{Segunda fase}

\section{Distribución del grupo clase por equipos}

Garantizar la heterogeneidad en los grupos de trabajo es fundamental. De esta forma se garantiza que haya distintas miradas y potencialidades en cada uno. El objetivo es que ellos se vayan convirtiendo en equipos. Un equipo es una forma de organización particular del trabajo, donde se busca que aflore el talento colectivo y la energía de las personas (Gómez y Acosta, 2003). Esta forma de organización es particularmente útil para alcanzar altos niveles de calidad en la gestión de una entidad. Para promover esta transición se les pide que realicen una alianza, un documento donde explicitan cómo funcionará el equipo, qué aportará cada estudiante al resto del equipo y qué necesitan para avanzar con mayor eficacia.

\section{Tercera fase}

\section{Trabajo colaborativo}

Es el momento de poner en marcha la actividad y es cuando los estudiantes empiezan a buscar información sobre las bibliotecas escolares. También cada miembro del equipo explica al resto del equipo la biblioteca del centro escolar donde realiza las prácticas y reflexionan sobre sus virtudes y oportunidades. El equipo debe escoger una experiencia de 
biblioteca escolar y trabajar sobre ella.

La elaboración del trabajo escrito se realiza utilizando las tecnologías a través del Google Drive dando respuesta a la cuestión planteada. Este Google Drive lo comparten con el profesor para que éste, en caso que sea necesario, les realice feedback para producir unos resultados óptimos. Con este retorno se garantiza que los estudiantes realicen la tarea bajo una perspectiva constructivista, entre todos crean el conocimiento, y añaden contenido que pueda faltar al ya existente. El trabajo avanza tras discusiones y correcciones que se realizan entre los estudiantes, con el propósito de obtener un buen resultado. Los estudiantes deben de autogestionarse según la alianza que determinaron en la segunda fase de esta actividad.

\section{Cuarta fase}

\section{Intercambio de trabajos}

En cuanto el trabajo escrito está finalizado se intercambian los trabajos entre los equipos. Se les instruye sobre cómo tratar el documento que les llega: tratarlo como documento autosuficiente, evitando todo tipo de personalismos y consultas externas, es decir, considerar el documento como la única evidencia del trabajo realizado por otro equipo, y solo pueden trabajar con lo que está escrito, evitando preguntar a los autores cualquier duda que tengan. Los equipos han de leer el documento e identificar el propósito de este para poder presentarlo. A través de este proceso, cuando les surgen interrogantes deben solucionarlos buscando información e imaginado distintos escenarios. Estos añadidos también deben ser expuestos en la exposición del trabajo. Los estudiantes aprenden del trabajo que han de presentar tanto por la forma como por el contenido que presenta.

Por lo que hace a la forma, los estudiantes toman conciencia del cómo se ha escrito el documento, qué estructura tiene y proponen también mejoras y ayudas para los autores del documento. Por lo que hace al contenido identifican qué elementos son significativos en lo que se presenta y qué le falta al documento, y qué aportaciones han de hacer para mejorar y seguir avanzando en él.

\section{Quinta fase}

\section{Co-presentación}

En esta fase los estudiantes presentan los trabajos que han revisado y preparado para ser expuestos. Cada equipo tiene la libertad de usar el método más adecuado para su presentación. El profesorado también ha trabajado con los documentos previamente al día de las exposiciones. Cada equipo realiza las presentaciones tan bien como sabe. El respeto y preparación de la presentación de un trabajo que no es propio formaliza este proceso, siendo la prudencia y la cautela elementos fundamentales de este proceso.

\section{Sexta fase}

\section{Valoración del trabajo escrito y del trabajo oral}

Esta fase consiste en valorar los trabajos que ha realizado cada equipo, tanto los de forma escrito como los de forma oral. Este proceso se desarrolla a través del método del feedforward. Es un proceso introspectivo que ayuda a la persona evaluada tomar conciencia de qué elementos puede mejorar y en cuales se compromete y cómo se prepara para mejorarlos.

La secuencia de este proceso tiene los siguientes pasos:

\section{Por parte del equipo que presenta oralmente:}

- Enumeración y evidencia de tres aspectos de las que están satisfechos tras su presentación

- Enumeración y evidencia de tres aspectos que se podrían mejorar y cómo lo podrían lograr

\section{Por parte de los observadores y grupo-clase:}

- Enumeración y evidencia de tres aspectos que se han realizado bien

- Enumeración y evidencia de tres aspectos que podrían mejorar y cómo lo podrían lograr

\section{Por parte del equipo evaluado por su trabajo escrito:}

- Compromiso de mejorar en las próximas entregas en una cuestión concreta y especificación de cómo lo realizarán.

Figura 2. Pasos para la realización del feedforward grupal. Fuente: elaboración propia.

Tras la sesión de presentación y feedforward cada equipo sabe cuáles son sus fortalezas y cómo puede seguir avanzando para mejorar y perfeccionar sus trabajos tanto de forma oral como de forma escrita.

Para finalizar la actividad, se revisa todo lo que ha sucedido con todo el grupo-clase. Por un lado, se sistematizan todas las fases que se han seguido para que tomen conciencia de los procesos que han llevado a cabo metodológicamente y de los aprendizajes y las competencias que han trabajado en cada fase. Y por el otro lado, se revisa si se han cumplido los objetivos que se pretendían en la actividad, valorándolo numéricamente de 0 a 10 para ver en qué medida se han asumido y cómo se quería avanzar. 


\section{Discusión y Conclusiones}

E l trabajo por competencias tal como las propone el EEES requiere de metodologías docentes nuevas que activen a los estudiantes (Zabalza y Arnau, 2007). El proceso formativo se entiende como bidireccional en el que los estudiantes trabajan, reflexionan, construyen y se responsabilizan de sus aprendizajes. El profesorado en esta situación desarrolla un rol didáctico distinto al tradicional: acompaña a los estudiantes a construir, reflexionar, actuar y responsabilizarse. De este modo se da a los estudiantes espacio para informarse, tomar conciencia, implicarse, decidir, comprometerse con y responsabilizarse de su proceso de aprendizaje. De esta forma el rol del estudiante también cambia sustancialmente: actuando para aprender y tomando parte de los proceso formativo basados en competencias. El estudiante, desde estas metodologías, trabaja por su desempeño profesional, viéndose así preparado para adaptarse a las incertidumbres y ambigüedades del mercado laboral.

Así que para que este proceso metodológico activo de enseñanza-aprendizaje sea posible es imprescindible un cambio de percepción tanto por parte del profesorado como del estudiante acostumbrados a metodologías más unidireccionales, centradas en conocimientos y no tanto en saberes competenciales. Esto requiere de mayor esfuerzo, paciencia, dedicación, acompañamiento y mentorización por parte de todos los actores implicados.

Una metodología activa necesita de actividades dinámicas en el aula, que interpelan continuamente a profesores y estudiantes. Los primeros por cuestionar sus saberes

${ }^{66}$ Para que este proceso
metodológico activo de
enseñanza-aprendizaje sea
posible es imprescindible
un cambio de percepción
tanto por parte del
profesorado como del
estudiante acostumbrados
a metodologías más
unidireccionales, centradas
en conocimientos y no tanto
en saberes competenciales."

científicos mediante la bidireccionalidad continua en el aula con los estudiantes, que complementan sus argumentaciones con los saberes que se encuentran en las tecnologías de la información y de la comunicación. Los segundos, por la necesidad de construir conocimiento científico y fundamentado teóricamente.

Las propuestas educativas activas y participativas, como por ejemplo la del feedforward, han de poder ayudar a los estudiantes a saber qué hacen bien y qué pueden mejorar, y planificar cómo lo harán; han de ayudarles a conocerse a sí mismos para poder percibir su genuinidad, en el que la toma de consciencia de sí mismo, la conciencia social, la gestión de las relaciones y la autogestión (Goleman, 2016) sean pilares fundamentales en su quehacer profesional.

A través de su proceso cooperativo y reflexivo, la actividad de co-presentación obliga a los estudiantes a:

1 Analizar la realidad de las bibliotecas escolares a través de las prácticas, estudiando sus posibilidades y dificultades que pueden presentar para los niños del hoy y del mañana;

2 Deconstruir y construir un contenido fundamentado teóricamente mediante el diálogo y consenso, la crítica y autocrítica entre los integrantes del equipo;

3 Trabajar en equipo creando una alianza, gestionando y tomando consciencia de los distintos liderazgos que hay, y analizando qué estilo de liderazgo es el que se define en cada uno;

4 Estudiar y valorar un documento de otro equipo, estudiar sus características y potencialidades y sugerir posibles mejoras;

5 Presentar el trabajo del otro equipo desde la responsabilidad pedagógica contribuyendo a su mejora y avance profesional;

6 Recibir las críticas de los compañeros con el propósito de mejorar y convertirse, si quieren, en excelentes profesionales.

La sistematización de esta propuesta formativa activa podría contribuir a que sea transferible a otras materias siempre y cuando se quieran tratar este conjunto de competencias. $\mathrm{Su}$ adaptabilidad dependerá del reto que se proponga en su inicio y definirá los contenidos a trabajar. Así pues, el elemento que cabe definir muy bien es el reto que se plantea a los estudiantes para adaptarse a la materia. Este debe de ser lo suficiente interesante y realista como para hacer que se sienten interpelados, motivados y entregados y a la vez con un nivel de dificultad suficiente como para que se sientan responsables de indagar, crear nuevas estructuras mentales y físicas, evaluar, discutir y reflexionar sobre la materia, mediante las revisiones bibliográficas y de contenido de la asignatura. 


\section{Referencias bibliográficas}

Álvarez Rateike, J. (2011). Formación académica vs. formación práctica: la eterna disputa. Obtenido de Educaweb Monográfico, 240. Recuperado en 14 de noviembre de 2019, de https://www.educaweb.com/ noticia/2011/10/31/formacion-academica-vs-formacionpractica-eterna-disputa-5046/

https://doi.org/10.30827/profesorado.v23i3.9302

Bauman, Z. (2007). Los retos de la educación en la modernidad líquida. Barcelona: Editorial Gedisa.

https://doi.org/10.14409/ie.v1i5.3947

European Ministers Responsible for Higher Education (2009). The Bologna Process 2020-The European Higher Education Area in the new decade”. Leuven and Louvain-la-Neuve, 28-29 April.

Borrás, I. (1997). Enseñanza y aprendizaje con la Internet: una aproximación crítica. Pixel-Bit, 9, 5-13.

Cano, E. (2008). La evaluación por competencias en la educación superior. Profesorado: revista de currículum y formación del profesorado, 12(3), 1-16.

https://doi.org/10.30827/profesorado.v22i3.8006

Cochran-Smith, M. (2002). Learning and unlearning: The education of teacher educators. Teachers and Teacher Education, 19, 5-28.

https://doi.org/10.1016/s0742-051x(02)00091-4

Collet-Sabé, J. (2011). Educació:¿arte, burocracia o artesania? Por una nueva metàfora de la teoría y de la práctica educativa. Pedagogia i treball social. Revista de Ciències Socials Aplicades 1(1), 27-50.

Estella, A. M., y Vera, C. S. (2008). La enseñanza en competencias en el marco de la educación a lo largo de la vida y la sociedad del conocimiento. Revista iberoamericana de educación, 47, 159-183.

https://doi.org/10.35362/rie5531589

Gaitán, L. (2010). Ser niño en el siglo XXI. Cuadernos de pedagogía, 407, 12-17.

Gibbs, G. y Simpson, C. (2009). Condiciones para una evaluación continuada favorecedora del aprendizaje. Barcelona: Octaedro.

Goldsmith, M. (2003). Try feedforward instead of feedback. Journal for Quality and Participation, 26(3), 38-40.

Goleman, D. (2016). Liderazgo. El poder de la inteligencia emocional. Barcelona: EdicionesSB.
Gómez Mujica, A. Y Acosta Rodríguez, H. (2003). Acerca del trabajo en grupos o equipos. Acimed, 11(6), Recuperado en 14 de noviembre de 2019, de http://scielo.sld.cu/scielo. php?script=sci arttext\&pid=S1024-94352003000600011\&lng =es\&tlng=es.

Graell, M. y Fuertes, M.T. (2019). Del Maestro experto al maestro novel: una experiencia de aps. En M. Raposo, O. Canet, M.D.Cebrián de la Serna, M.A. Barberá, G. A. Pérez Abellás, M.A. Zabalza Beraza, XV Symposium Internacional sobre el Prácticum y las Prácticas Externas "Presente y retos de futuro" (pp.209-220). Pontevedra: Asociación para el prácticum y de las prácticas externas. https://doi.org/10.36576/summa.14172

Imbernon Muñoz, F., y Medina Moya, J. L. (2005). Metodología participativa en el aula universitaria. La participación del alumnado. Cuadernos de docencia universitaria, 4. Recuperado en 14 de noviembre de 2019, de http://diposit.ub.edu/dspace/bitstream/2445/1041/1/163. pdf https://doi.org/10.14409/au.v0i19.6793

Marcelo, C. (2009). Desarrollo profesional docente: pasado y futuro. Sisifo. Revista de Ciencias de la Educación, 7, $7-22$.

Martín y Puig, (2007) Las siete competencias básicas para educar en valores. Barcelona: Graó.

Martínez, F., y Prendes, M. (2007). Nuevas tecnologías y educación. Estimado colega. Madrid: Pearson educación. 27-33.

Miller, G. (1990). The assessment of Clinical Skill. Compotence-Performance. Academic Medicine, 9 (65), 63-67.

Myers, E.D. (1960) Education in the perspective of History. New York: Harper.

Prendes, M. P. (2003). Aprendemos--¿cooperando o colaborando?. Las claves del método. En F. Martínez (coord.) Redes de comunicación en la enseñanza: las nuevas perspectivas del trabajo corporativo. Barcelona: Paidós Ibérica. (pp. 93-127).

https://doi.org/10.35376/10324/21465

Perrenoud, P. (2004). Diez nuevas competencias para enseñar. Barcelona: Graó.

Pujolàs, P., Lago, J. R., Naranjo, M., Pedragosa, O., Riera, G., Soldevila, J. y Rodrigo, C. (2011). El programa CA/ AC ("cooperar para aprender/aprender a cooperar") para enseñar a aprender en equipo. Implementación del aprendizaje cooperativo en el aula. Barcelona: 
Universitat Central de Catalunya.

https://doi.org/10.21158/9789587560015

Schön, D. (1992). La formación de los profesionales reflexivos. Hacia un nuevo diseño de la enseñanza y el aprendizaje en las profesiones. Barcelona: Paidós-Mec.

Álvarez Suarez, Alberto; González García, Julio Antonio; Alonso Álvarez, Javier y Arias Pérez, Jorge L. (2014). Indicadores centinela para el plan de Bolonia. Revista de Investigación Educativa, 32(2), 327-338.

https://doi.org/10.6018/rie.32.2.171751

Trilla, J. (2009) El aprendizaje servicio y necesidades sociales. En J.M. Puig (coord.) Aprendizaje servicio (ApS) Educación y compromiso cívico. Barcelona: Graó, 33-51

Zabala, Z. y Arnau, L. (2007). 11 Ideas Clave. Cómo aprender y enseñar competencias. Barcelona: Graó. 


\section{Annexos}

\section{Annexo 1}

\section{Competencias Generales (CG):}

CG-04 Promover y facilitar en los contextos de aprendizaje y de convivencia, en la escuela y en relación con el entorno, la atención a las necesidades educativas singulares de los estudiantes, a la igualdad de género, a la equidad y al respeto a los derechos humanos.

CG-07 Conocer los elementos que conforman el pensamiento crítico, los distintos niveles de dominio, y desarrollar esta competencia al máximo.

CG-08 Conocer los elementos que conforman el pensamiento reflexivo, los distintos niveles de dominio, y desarrollar esta competencia al máximo.

CG-09 Conocer los elementos que conforman la competencia de la creatividad, los distintos niveles de dominio, y desarrollar esta competencia al máximo.

CG-10 Conocer los elementos que conforman la competencia de espíritu emprendedor, los distintos niveles de dominio, y desarrollar esta competencia al máximo.

\section{Competencias específicas de titulación (CET):}

CET-2 Responsabilidad pedagógica: poseer los conocimientos y las habilidades pedagógicas para crear un entorno de aprendizaje seguro, para toda una clase o para un grupo, pero también para alumnos individuales, y dar lugar a que los niños puedan convertirse en adultos responsables y autónomos.

CET-4 Responsabilidad organizativa: poseer un conocimiento y unas habilidades organizativas suficientes para crear un clima de trabajo agradable en sus clases y en la clase: organizado, ordenado y centrado en todos los aspectos, con él mismo y con sus compañeros, y por encima de todo claro con sus alumnos y lograr todo esto de una manera profesional y sistemática.

CET-5 Colaboración con los compañeros de profesión: poseer los conocimientos y las habilidades necesarios para realizar una aportación profesional al clima pedagógico de la escuela, así como a las relaciones profesionales, y con el fin de lograr una buena organización escolar.

CET-7 Fomento de la responsabilidad en lo que se refiere al desarrollo profesional: analizar, reflexionar y desarrollar los puntos de vista sobre la profesión y la competencia como maestro, saber explicitarlos y saber cómo ponerse al día.

\section{Competencias específicas de materia (CEM):}

CEM-42 Conocer el currículo de lengua y lectoescritura de esta etapa así como las teorías sobre la adquisición y el desarrollo de los aprendizajes correspondientes.

CEM-43 Favorecer las capacidades de habla y de escritura.

CEM-44 Conocer y dominar técnicas de expresión oral y escrita.

CEM-45 Conocer la tradición oral y el folclore.

CEM-46 Comprender el paso de la oralidad a la escritura y conocer los diferentes registros y usos de la lengua.
CEM-47 Conocer el proceso de aprendizaje de la lectura y la escritura y su enseñanza.

CEM-48 Afrontar situaciones de aprendizaje de lenguas en contextos multilingües.

CEM-49 Reconocer y valorar el uso adecuado del lenguaje verbal y no verbal.

CEM-50 Conocer y utilizar adecuadamente recursos para el fomento de la lectura y la escritura.

CEM-51 Adquirir formación literaria y, en especial, conocer la literatura infantil.

Annexo 2

\section{Competencias específicas de materia:}

CEM-43 Favorecer las capacidades de habla y de escritura.

CEM-50 Conocer y utilizar adecuadamente recursos para el fomento de la lectura y la escritura.

CET-2 Responsabilidad pedagógica: poseer los conocimientos y las habilidades pedagógicas para crear un entorno de aprendizaje seguro, para toda una clase o para un grupo, pero también para alumnos individuales, y dar lugar a que los niños puedan convertirse en adultos responsables y autónomos.

CG-07 Conocer los elementos que conforman el pensamiento crítico, los distintos niveles de dominio, y desarrollar esta competencia al máximo.

CG-08 Conocer los elementos que conforman el pensamiento reflexivo, los distintos niveles de dominio, y desarrollar esta competencia al máximo. 